Original Research

\title{
Monocytes and pyrophosphate promote mesenchymal stem cell viability and early osteogenic differentiation
}

\author{
Sara Svensson ${ }^{1} \cdot$ Michael Palmer $\mathbb{1}^{2} \cdot$ Johan Svensson ${ }^{3} \cdot$ Anna Johansson $\mathbb{B}^{1} \cdot$ Håkan Engqvist $\mathbb{D}^{2} \cdot$ Omar Omar $\mathbb{D}^{4} \cdot$ \\ Peter Thomsen (10)
}

Received: 26 June 2021 / Accepted: 18 December 2021 / Published online: 15 January 2022

(c) The Author(s) 2022

\begin{abstract}
Pyrophosphate-containing calcium phosphate implants promote osteoinduction and bone regeneration. The role of pyrophosphate for inflammatory cell-mesenchymal stem cell (MSC) cross-talk during osteogenesis is not known. In the present work, the effects of lipopolysaccharide (LPS) and pyrophosphate (PPi) on primary human monocytes and on osteogenic gene expression in human adipose-derived MSCs were evaluated in vitro, using conditioned media transfer as well as direct effect systems. Direct exposure to pyrophosphate increased nonadherent monocyte survival (by $120 \%$ without LPS and 235\% with LPS) and MSC viability (LDH) (by 16-19\% with and without LPS). Conditioned media from LPSprimed monocytes significantly upregulated osteogenic genes (ALP and RUNX2) and downregulated adipogenic (PPAR- $\gamma$ ) and chondrogenic (SOX9) genes in recipient MSCs. Moreover, the inclusion of PPi $(250 \mu \mathrm{M})$ resulted in a 1.2- to 2-fold significant downregulation of SOX9 in the recipient MSCs, irrespective of LPS stimulation or culture media type. These results indicate that conditioned media from LPS-stimulated inflammatory monocytes potentiates the early MSCs commitment towards the osteogenic lineage and that direct pyrophosphate exposure to MSCs can promote their viability and reduce their chondrogenic gene expression. These results are the first to show that pyrophosphate can act as a survival factor for both human MSCs and primary monocytes and can influence the early MSC gene expression.
\end{abstract}

\section{Graphical abstract}

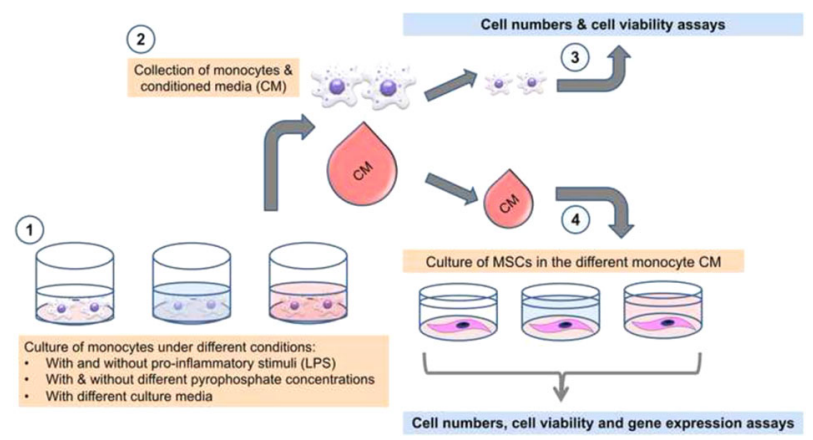

Supplementary information The online version contains supplementary material available at https://doi.org/10.1007/s10856021-06639-y.

Peter Thomsen

peter.thomsen@biomaterials.gu.se

1 Department of Biomaterials, Institute of Clinical Sciences, Sahlgrenska Academy, University of Gothenburg,

Gothenburg, Sweden

2 Department of Engineering Sciences, Applied Materials Science Section, Uppsala University, Uppsala, Sweden
3 Department of Statistics, Umeå School of Business, Economics and Statistics, Umeå University, Umeå, Sweden

4 Department of Biomedical Dental Sciences, College of Dentistry, Imam Abdulrahman bin Faisal University, Dammam, Saudi Arabia 
Keywords Cell-cell communication $\cdot$ Gene expression $\cdot$ Monocytes $\cdot$ MSC $\cdot$ Osteoinduction

\section{Introduction}

Bone tissue has a remarkable ability to regenerate following injury. However, malunion or nonunion still occurs in more than $10 \%$ of traumatic injury cases [1], especially when the degree of injury exceeds the body's ability to repair or when the healing is compromised. Currently, the most promising pro-regenerative tools for bridging large defects and improving the rate of bone regeneration are materials (i.e., tissue scaffolds or granules) and biochemical signals that stimulate the recruitment and/or differentiation of stem cells and osteoprogenitors (e.g., growth factors, such as BMP-2, or bioactive ions, such as calcium and strontium) [2, 3]. The bone regenerative effects of such natural and synthetic cues are commonly judged by the response of osteoblastic cell lines in vitro or the degree of bone formation in vivo. On the other hand, the role of other cell types and biological processes, such as immune cells and inflammation, in mediating potential material effects remains much less explored.

Inflammatory cells play a major role in bone tissue healing and regeneration after trauma. Circulating monocytes are actively recruited to an injury site $[1,4]$ where they differentiate into macrophages that recruit and activate multiple cell types. Monocytes/macrophages express and secrete biological mediators that recruit mesenchymal stem cells (MSCs) [5] and osteoblasts [6]. Moreover, progenitors from the monocytic lineage can directly differentiate into bone-resorbing osteoclasts [6] and can also polarize towards pro-inflammatory (M1) or pro-regenerative (M2) macrophage phenotypes [7-9]. In addition to their role during the acute healing phase, it has been suggested that signals secreted by macrophages modulate the regenerative process even after the initial inflammatory response is resolved $[8,9]$. It has been shown in vitro that conditioned media (CM) from macrophages cultured on smooth and microporous titanium surfaces, under different stimulation conditions, convey differential osteogenic effects on recipient MSCs [10]. Interestingly, the modest effect of the surface properties communicated by macrophages was surpassed by the classical activation of M1 macrophages by lipopolysaccharide (LPS) [10]. Specifically, proinflammatory M1 macrophages induced osteogenic gene expression in recipient MSCs, but IL-4-stimulated (M2) macrophages did not [10]. In contrast, another study concluded that materialinduced M2 macrophages stimulate the proliferation and differentiation of MSCs, leading to improved vascularization and angiogenic gene expression [11].

In vitro studies have suggested that specific phases of calcium phosphates promote inflammatory, immunomodulatory and osteogenic responses from monocytes/macrophages and/ or MSCs [11-13]. Calcium pyrophosphate, an insoluble calcium phosphate that elicits a strong inflammatory response $[13,14]$, has been shown to stimulate osteogenic gene expression in vitro [15] and bone formation in vivo $[16,17]$. With respect to inflammatory cells, pyrophosphate crystals induce potent expression and secretion of proinflammatory cytokines (IL-1 $\beta$, IL-6, and IL-8) via activation of the MAPKdependent NF-kB pathway [13]. The same pathway is well recognized to mediate LPS-induced expression and secretion of major proinflammatory cytokines by macrophages [18, 19]. Interestingly, inflammatory cells can also directly regulate endogenous pyrophosphate production and localization via proinflammatory cytokine-mediated expression of the pyrophosphate generating, transporting and digesting (phosphoesterase) proteins ENPP1, ANKH, and ENT 1 [20, 21]. However, it is unclear whether the macrophage response is affected by pyrophosphate concentration and whether the pyrophosphate-induced effects on macrophages are influenced by the presence of proinflammatory mediators, such as LPS.

Pyrophosphate ions are naturally transported out of osteoblasts and osteogenic progenitors (MSCs) to regulate mineralization and prevent pathological calcification of the extracellular matrix [20, 22, 23]. In contrast, when osteogenic cells encounter pyrophosphate-containing surfaces (i.e., coatings or cell scaffolds), their response is assumed to differ from that observed when contacting naturally formed nano- and micron-scale precipitants, which are known to elicit a vigorous inflammatory condition in joints (i.e., calcium pyrophosphate deposition (CPPD) in pseudogout) $[24,25]$. Instead, pyrophosphate-containing materials [17] and coatings $[26,27]$ have been shown to promote osteogenic effects. Recently, pyrophosphate-containing bioceramics were shown to considerably promote bone regeneration in osseous and nonosseous sites [28]. Histological evidence revealed that the pyrophosphate-containing bioceramic induces the differentiation of MSCs to osteoblastic cells in parallel to macrophage accumulation in the vicinity of the implant and bone-forming osteoblasts. Therefore, it is important to explore whether exposure to pyrophosphate has a direct effect on MSCs or whether the pyrophosphate effect on MSCs is indirectly mediated via macrophages.

When evaluating biomaterials, although single cell type in vitro assays provide simple screening systems, they are far from representing the complex in vivo milieu [29]. Attempts have been made to mimic the 3-dimensional in vivo healing environment around implants, using, for instance, explanted [30] or tissue-engineered [31] bone in 
combination with regenerative stem cells. Moreover, conditioned media $(\mathrm{CM})$ transfer $[10,32]$ and co-culture $[33,34]$ in vitro studies have provided mechanistic insight into the role of cell-cell communication in the cellular response to the material properties. Soluble factors and signals conveyed by inflammatory cells to stem cells are of particular interest.

In the present study, the effects of LPS and disodium pyrophosphate $(\mathrm{PPi})$ on primary human monocytes and osteogenic gene expression in human adipose-derived MSCs were evaluated. The aim was to determine whether different concentrations of pyrophosphate influence macrophage and MSC responses and whether the priming of MSCs with LPS or LPS-treated monocyte conditioned medium enhances osteogenic gene expression in MSCs. The null-hypothesis is that LPS and/or PPi neither influence the human monocyte cell number and viability nor convey any direct or indirect effects on cell number, viability and early osteogenic gene expression of human MSCs.

\section{Materials and methods}

\subsection{Pyrophosphate}

A $50 \mathrm{mM}$ sodium pyrophosphate stock solution was prepared by dissolving sodium pyrophosphate $\left(\mathrm{H}_{2} \mathrm{Na}_{2} \mathrm{O}_{7} \mathrm{P}_{2}\right.$; Sigma-Aldrich, Steinheim, Germany) in deionized water, neutralizing with $2.0 \mathrm{M}$ sodium hydroxide $(\mathrm{NaOH})$ and passing through a $0.22 \mu \mathrm{m}$ sterile filter. The stock solution was prepared fresh prior to each treatment.

\subsection{Cell culture}

Buffy coats were purchased from the Blood Central (Sahlgrenska University Hospital, Sweden). The buffy coats were received after deidentification with respect to name, gender and age by the Blood Central. Primary human monocytes were isolated from buffy coats of five anonymous healthy blood donors using Ficoll separation followed by negative selection on a magnetic column (MACS; Miltenyi Biotec, Bergisch Gadbach, Germany; viability >98\%). May-Grünewald Giemsa-stained cytospin slides showed $97.4 \pm 0.3 \%$ monocyte purity, with lymphocytes being the primary source of contamination. Monocytes were seeded into tissue culturetreated 6-well polystyrene plates (Falcon ${ }^{\mathrm{TM}}$, BD Biosciences, San Jose, CA, USA) at a concentration of $0.5 \times 10^{6}$ cells $/ \mathrm{mL}$ in a total of $5 \mathrm{~mL}$ Dulbecco's modified Eagle's medium-low glucose (DMEM-LG; Lonza, Bornem, Belgium) supplemented with $10 \%$ fetal bovine serum (Gibco, UK), $1 \%$ L-glutamine ( $2 \mathrm{mM}$; Gibco) and $1 \%$ pencillin/streptomycin (PEST; Sigma-Aldrich, Saint Louis, MO, USA). After overnight incubation $\left(37^{\circ} \mathrm{C}\right.$, humidified atmosphere with 5\%
$\mathrm{CO}_{2}$ ), the medium was exchanged to a low-serum variant $(1 \%)$, and the cells were treated with the following concentrations of pyrophosphate (PPi): $0 \mu \mathrm{M}$ (PPi0), $50 \mu \mathrm{M}$ (PPi1), $100 \mu \mathrm{M}$ (PPi2) or $250 \mu \mathrm{M}$ (PPi3) with or without $10 \mathrm{ng} / \mathrm{mL}$ lipopolysaccharide (LPS; Sigma-Aldrich), yielding a total of eight different environments (two wells per environment). The cells were incubated in the medium for $48 \mathrm{~h}$, yielding monocyte-conditioned medium (CM). In parallel, control medium (CtrM) was produced by incubating in an identical set of 6-well plates using the same stimulatory conditions but without cells. In addition, medium to be used for direct stimulation of MSCs was placed in a flask in the incubator, later yielding direct effect medium (DEM). Monocyte-CM and CtrM were transferred into $15 \mathrm{~mL}$ tubes and centrifuged $(500 \mathrm{~g}, 10 \mathrm{~min})$, after which the supernatants from the same conditions were pooled (total $10 \mathrm{~mL}$ ).

Human adipose-derived mesenchymal stem cells (ATCC \#63557082), passage 5, were seeded into tissue culturetreated 24-well polystyrene plates $\left(\right.$ Falcon $\left.^{\mathrm{TM}}\right)$ at a concentration of 36,000 cells $/ \mathrm{mL}$ in $1 \mathrm{~mL}$ expansion medium containing DMEM-LG supplemented with $10 \%$ FBS, $1 \%$ L-glutamine $(2 \mathrm{mM}), 1 \%$ PEST and $0.01 \%$ fibroblast growth factor (FGF; Life Technologies, UK). After $48 \mathrm{~h}$ of culture, the medium was exchanged with $\mathrm{CM}$, CtrM or DEM. The DEM wells were treated with the following PPi concentrations: $0 \mu \mathrm{M}$ (PPi0), $50 \mu \mathrm{M}$ (PPi1), $100 \mu \mathrm{M}$ (PPi2) or $250 \mu \mathrm{M}$ (PPi3) with or without $10 \mathrm{ng} / \mathrm{mL}$ LPS. After $72 \mathrm{~h}$ of incubation, the MSCs were harvested. The outline of the in vitro experiments is presented in Fig. 1.

\subsection{Cell number}

Quantification of adherent and supernatant monocytes and MSCs (analyzed in duplicate samples) was performed using the Nucleocounter system (ChemoMetec A/S, Allerød, Denmark). Briefly, equal amounts of lysing buffer A and stabilization buffer B (provided with the system) were added to each sample; vortexing was performed after each addition and prior to measurement. The samples were loaded in a Nucleocassette ${ }^{\mathrm{TM}}$ containing propidium iodide, and the cell nuclei were automatically counted. For viability measurements of purified monocytes, an additional cell sample without the addition of lysis buffer was analyzed to detect dead cells in the sample.

\subsection{Cell viability}

Cell viability was assessed in centrifuged medium (300 g, 5-10 min) in duplicate samples by analyzing the lactate dehydrogenase (LDH) content (C-laboratory, Sahlgrenska University Hospital, Gothenburg, Sweden). The detection limit of the instrument was $0.17 \mu \mathrm{Katal} / \mathrm{L}$. Values below the detection limit were set at $0.16 \mu \mathrm{Katal} / \mathrm{L}$. 
Fig. 1 Schematic drawing of the experimental outline. Monocytes isolated from buffy coats were seeded into 6-well plates and incubated overnight. The medium was changed, and monocytes were exposed to varying concentrations of pyrophosphate (PPi) in the presence or absence of LPS for $48 \mathrm{~h}$. The medium was withdrawn, centrifuged, and added to MSCs for $72 \mathrm{~h}$ prior to analysis. In parallel, control media (CtrM) - without monocytes - with the addition of $\mathrm{PPi}$ and LPS were incubated for $48 \mathrm{~h}$ before centrifugation and exposure to MSCs. In a third track, medium was incubated for $48 \mathrm{~h}$ before addition to MSCs with additional direct exposure of MSCs to PPi and LPS, referred to as direct effect media (DEM)

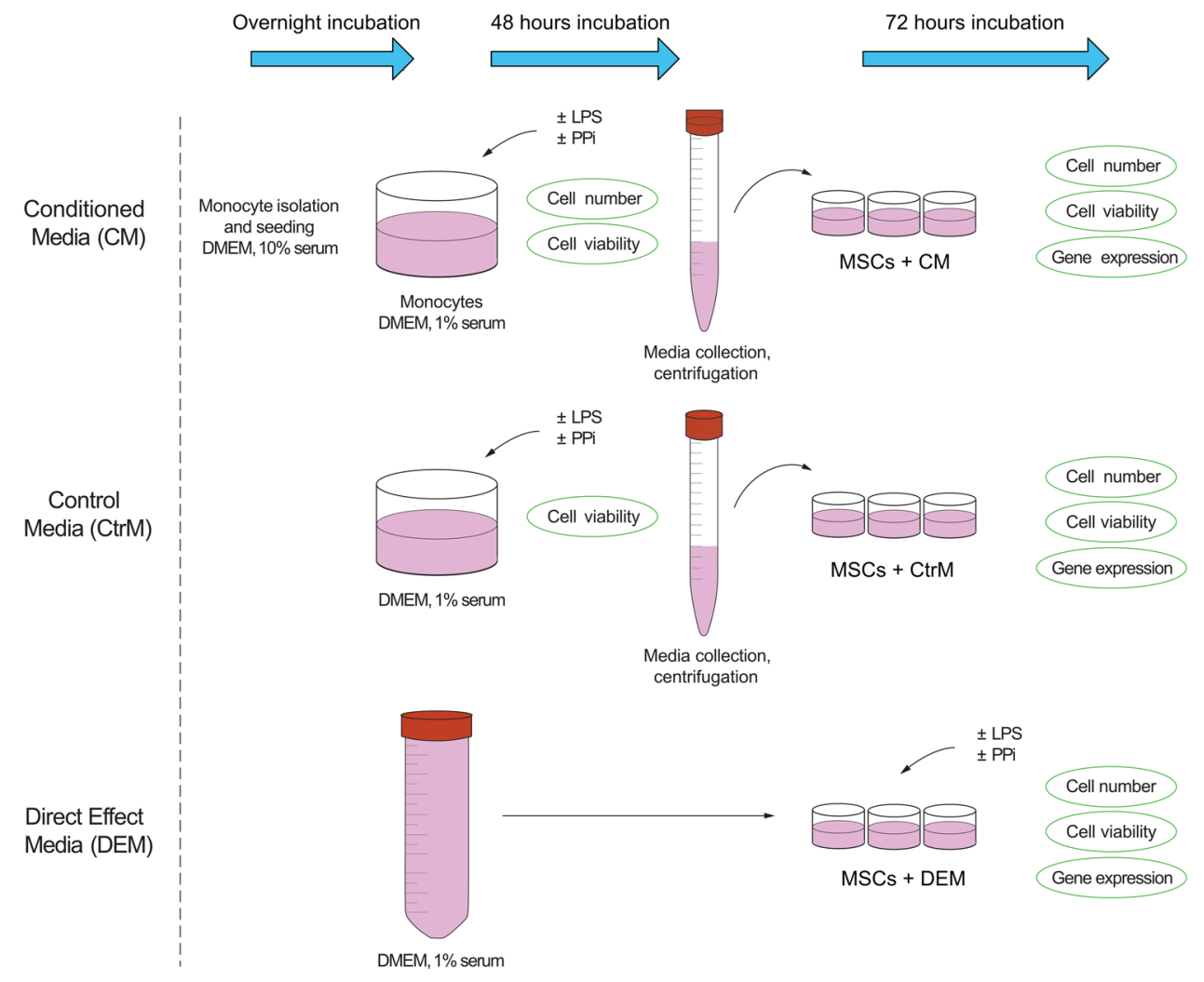

analyzed genes were related to the following: osteogenic differentiation: alkaline phosphatase (ALP), bone morphogenetic protein 2 (BMP-2), type I collagen alpha (COL1A1), runt-related transcription factor 2 (RUNX2), osteopontin $(O P N)$, transforming growth factor beta (TGF$\beta)$; cell proliferation: marker of proliferation Ki-67 (KI67), proliferating cell nuclear antigen (PCNA); cell death: caspase 3 (CASP3), tumor protein p53 (P53); and chondrogenic- or adipocyte differentiation: $S R Y$-box transcription factor 9 (SOX9) and peroxisome proliferator-activated receptor gamma (PPAR- $\gamma)$. Tyrosine 3-monooxygenase /tryptophan 5-monooxygenase activation protein zeta (YWHAZ) was identified as the best reference gene in the TATAA reference gene panel as provided in the Online Resource 1 (Supplementary Table S1) based on analysis in GenEx software version 6 (MultiD Analyses AB, Gothenburg, Sweden) using both the geNorm and NormFinder algorithms. Raw data were analyzed on LightCycler ${ }^{\circledR} 480$ Software, Version 1.5 (Roche Applied Science) and processed in GenEx using the relative comparative Cq method. ValidPrime (TATAA Biocenter AB) was used for the detection and correction of contaminating genomic DNA.

\subsection{Statistical modeling and analysis}

The experiment was analyzed in a series of MANOVAs in blocks of responses that were connected, i.e., bone-related 
gene expression (ALP, BMP-2, COL1A1, RUNX2, OPN and $T G F-\beta$ ), cartilage- or adipose-related gene expression ( $S O X 9$ and $P P A R-\gamma)$, proliferation-related gene expression (KI67, $P C N A)$, death-related gene expression (CASP3, P53), MSC cell numbers (adherent + supernatant cells) and viability, and monocyte cell numbers and viability. In MANOVAs related to MSCs, the model was defined by the factors LPS (with or without), material (PPi0, PPi1, PPi2, PPi3), the condition (CM, CtrM, DEM) and the interaction LPS $\times$ condition. In the MANOVA related to the monocytes, only the factors LPS and material were used. Individual factor-level effects were analyzed using estimated marginal means with Bonferroni correction. Further details on MANOVA results and associated complementary ANOVA analyses are found in the Online Resource 1 (Supplementary Table S2). Residual analyses were carried out to check the model assumptions. If the assumption of homoelasticity was doubtful, a sensitivity analysis using bootstrapping was carried out. The data were statistically evaluated in SPSS Statistics software 24.0 (IBM Corporation, Armonk, NY, USA), and a statistical significance level of 5\% was used. Bar graphs represent the means \pm standard error of the mean (SE).

\section{Results}

\subsection{Effects of LPS and PPi on monocyte adhesion/ cell numbers and viability}

Monocyte adhesion was increased by the presence of LPS $(P<0.001)$ but was not affected by PPi (Fig. 2a;

(a) Adherent monocytes

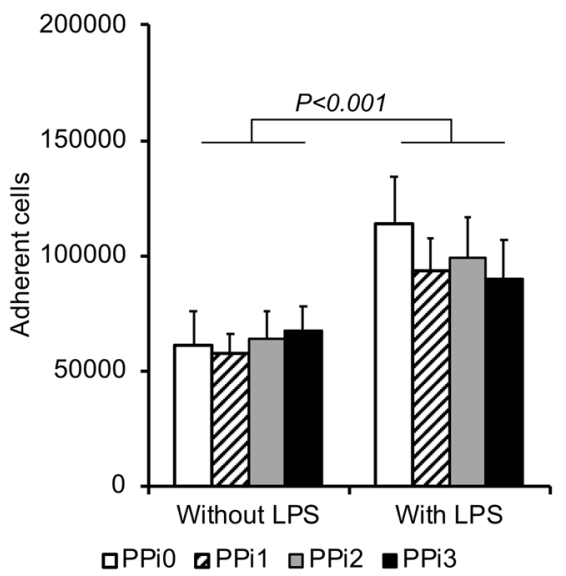

Fig. 2 Cell count of monocytes. Cell count of adherent monocytes (a) and monocytes in the culture media/supernatant (b). Number of monocytes after $48 \mathrm{~h}$ of culture in polystyrene plates in the presence or absence of lipopolysaccharide (LPS) and exposed to different concentrations of pyrophosphate (PPi): $0 \mu \mathrm{M}$ (PPi0), $50 \mu \mathrm{M}$ (PPi1), $100 \mu \mathrm{M}$ (PPi2) and $250 \mu \mathrm{M}$ (PPi3). The bar graphs show the means
Supplementary Table S1). The number of monocytes in the supernatant was significantly reduced by LPS $(20-47 \%$ decrease, $P<0.05$ ) (Fig. 2b; Online Resource 1 (Supplementary Table S2)) in all PPi groups. For both inflammatory and noninflammatory conditions, the number of supernatant cells increased with increasing PPi concentration, with significant differences for both the PPi2 and PPi3 concentrations (100 and $250 \mu \mathrm{M})$ in comparison to cells in the PPi0 group, without the inclusion of PPi (Fig. 2b). Monocyte viability was high (average LDH $0.16 \pm 0.02 \mu \mathrm{Katal} / \mathrm{L}$ ) and was unaffected by the presence of either PPi or LPS (Online Resource 1 (Supplementary Table S2)).

\subsection{Effects of LPS, PPi and condition on MSC adhesion/cell numbers and viability}

The amount of adherent MSCs in the wells after $72 \mathrm{~h}$ of incubation in the assigned media was similar between all conditions and inflammatory status and was not affected by PPi (Online Resource 1 (Supplementary Table S2)). However, the number of nonadherent MSCs (making up approximately $10 \%$ of the total number of cells) was significantly reduced in the CM from LPS-stimulated monocytes versus CM from nonstimulated monocytes (74-79\% decrease, $p<0.001$ ) (Fig. 3; Online Resource 1 (Supplementary Table S2)).

The average MSC viability ranged from 0.33 to $0.64 \mu \mathrm{Katal} / \mathrm{L}$ for the different conditions with or without LPS, with the highest viability among cells in the monocyte-conditioned media with LPS and the lowest viability for MSCs in monocyte-conditioned media without

\section{(b) Monocytes in supernatant}

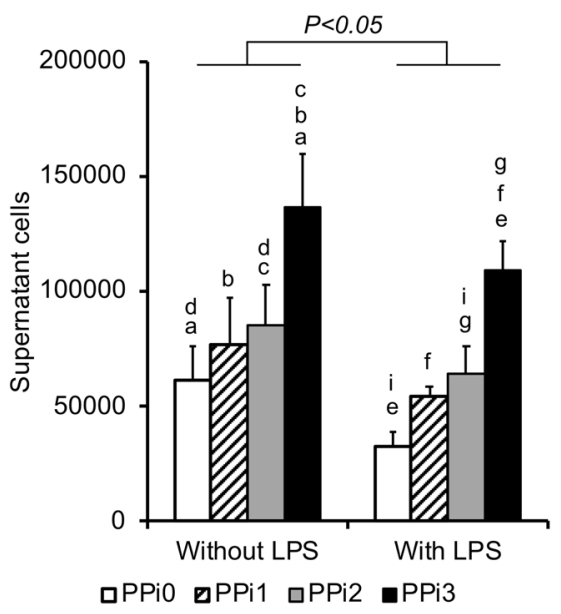

and standard error of the mean $(n=4-5)$. Connected bars indicate significant differences $(P<0.05 ; P<0.001)$ between LPS and nonLPS. Small letters indicate significant differences between PPi concentrations (PPi0, PPi1, PPi2, PPi3), where each two similar letters indicate a statistically significant difference $(P<0.05)$ between the two concentrations they are representing 


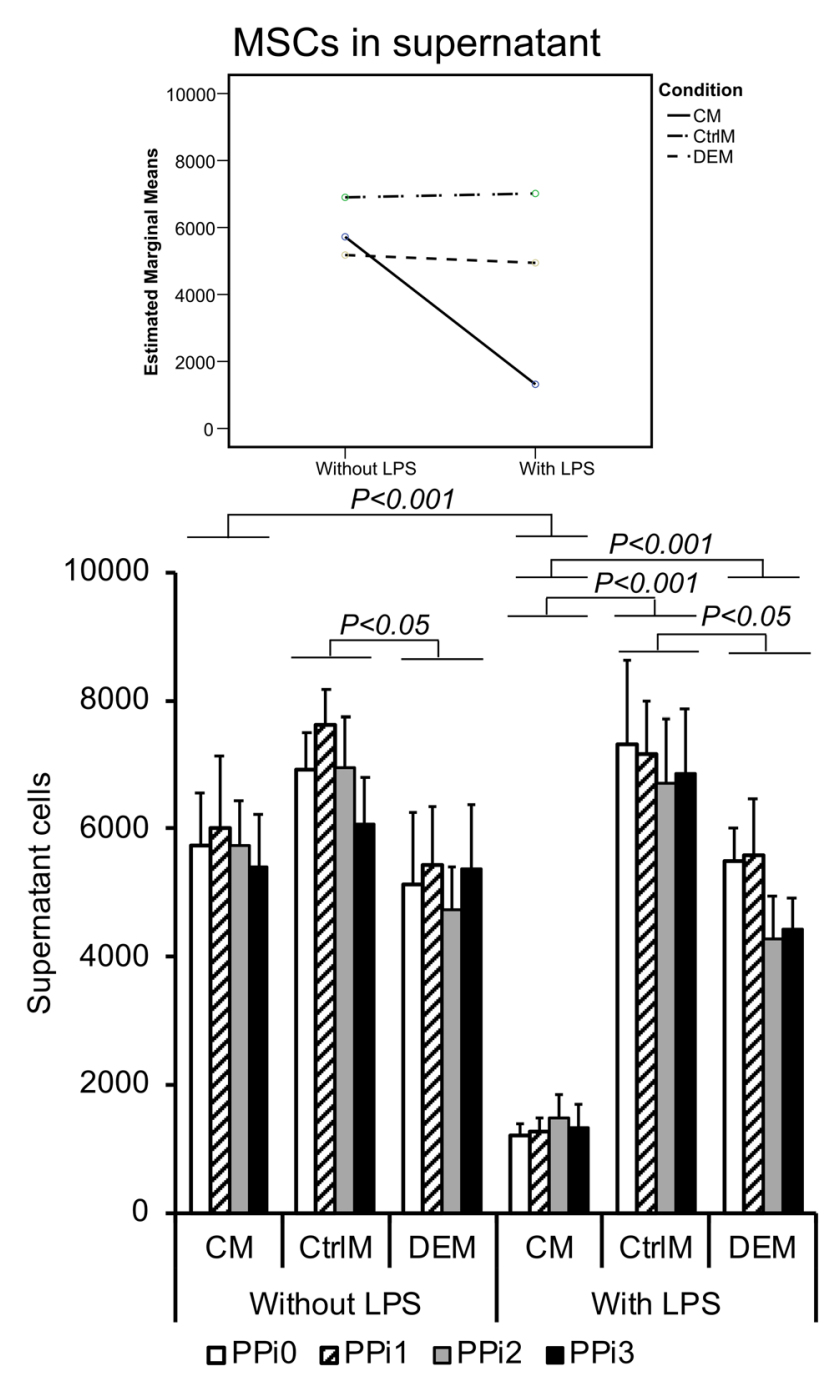

Fig. 3 Cell count of mesenchymal stem cells (MSCs). Number of MSCs in the supernatant after $72 \mathrm{~h}$ of culture in different culture media: $\mathrm{CM}=$ conditioned media from monocytes incubated for $48 \mathrm{~h}$ in polystyrene well plates in the presence or absence of lipopolysaccharide (LPS) and exposed to different pyrophosphate (PPi) concentrations; $\mathrm{CtrlM}=$ control media incubated for $48 \mathrm{~h}$ in polystyrene well plates in the presence or absence of LPS and exposed to different $\mathrm{PPi}$ concentrations; DEM $=$ direct effect media incubated for $48 \mathrm{~h}$ in $50 \mathrm{~mL}$ tubes and used for direct exposure of MSCs to LPS and different PPi concentrations. The different PPi concentrations were as follows: $0 \mu \mathrm{M}$ (PPi0), $50 \mu \mathrm{M}$ (PPi1), $100 \mu \mathrm{M}$ (PPi2) and $250 \mu \mathrm{M}$ (PPi3). The line graph (top) shows the interaction effect of culture condition and exposure to LPS, whereas the bar graph (bottom) shows the mean and standard error of the mean $(n=4-5)$. Connected bars indicate significant differences $(P<0.05 ; P<0.001)$ between LPS and non-LPS as well as between the different conditions (CM, CtrlM, DEM)

LPS. The highest concentration of PPi $(250 \mu \mathrm{M})$ resulted in significantly higher MSC viability compared with $0 \mu \mathrm{M} \mathrm{PPi}$ in all culture conditions (2-16\% without LPS and 10-9\% with LPS; $P<0.05$ across the groups), although this difference was more pronounced for cells in direct contact with PPi, i.e., DEM (Fig. 4).

\subsection{Effects of LPS, PPi and condition on MSC gene expression}

\subsubsection{Proliferation- and death-related genes}

MSC expression of the proliferation-related genes PCNA and KI67 and the cell death-related genes CASP3 and P53 did not show any dependence upon PPi concentration, inflammatory environment, or condition in the model used (Online Resource 1 (Supplementary Table S2)).

\subsubsection{Bone-related genes}

Following exposure to 24 different test conditions, distinct gene expression patterns were observed in MSCs. For bone-related genes, LPS exposure and condition, as well as the interaction effect between the two, were of importance, whereas the PPi concentration did not significantly affect gene expression (Online Resource 1 (Supplementary Table S2)). The key osteogenic commitment transcription factor $R U N X 2$ revealed 2.6- (PPi0), 2- (PPi1), 3- (PPi2) and 3.5-fold (PPi3) significantly higher expression in MSCs cultured in CM from LPSstimulated monocytes than in MSCs cultured in nonstimulated monocyte CM $(P<0.05$ across the material groups) (Fig. 5b). Similarly, the early osteogenic differentiation marker ALP showed 2.5- (PPi0), 3.5- (PPi1), 4(PPi2) and 5-fold (PPi3) significant upregulation in MSCs cultured in CM from LPS-stimulated monocytes compared to nonstimulated monocytes $(P<0.001$ across the material groups) (Fig. 5a). A similar pattern was seen for COL1A1 (Fig. 5c), although the effect of LPSstimulated monocyte $\mathrm{CM}$ did not reach statistical significance in the MANOVA test. In contrast, no major effect was observed for LPS-stimulated monocyte CM on the MSC expression of BMP-2 (Fig. 5d). For BMP2, a 2.5 -fold higher trend of expression was observed in MSCs directly exposed to the highest PPi concentration (PPi3) in the DEM (with and without LPS) compared to $\mathrm{PPi} 3$ in control media (CtrM) or to no-PPi (PPi0) in direct effect media (DEM) (Fig. 5d). Other bone-related genes, such as $O P N$ and $T G F-\beta$, were not affected by any of the investigated parameters $(P>0.05$, Online Resource 1 (Supplementary Table S2)).

\subsubsection{Cartilage- and adipose-related genes}

A significant downregulation in cartilage-related gene expression, $S O X 9$, was revealed when MSCs were exposed to inflammatory monocyte-conditioned media (Fig. 6a). Moreover, the presence of PPi resulted in downregulation of SOX9 gene expression in a concentration-dependent manner, with a significant difference between $\mathrm{PPi} 3$ and PPi0 


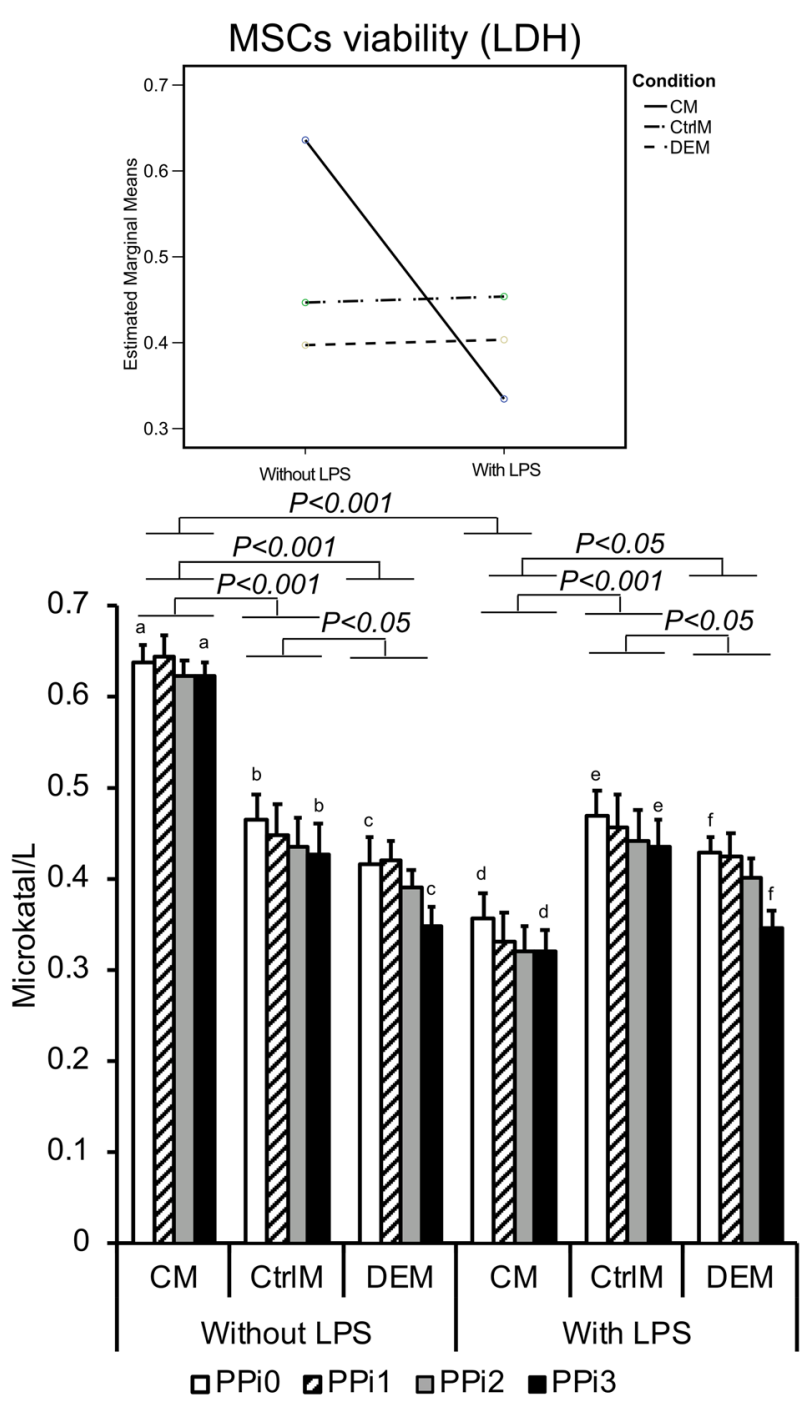

Fig. 4 Viability of mesenchymal stem cells (MSCs). The viability of MSCs was measured by lactate dehydrogenase (LDH) analysis. The LDH enzyme activity in the media of MSCs after $72 \mathrm{~h}$ of culture in different culture media: $\mathrm{CM}=$ conditioned media from monocytes incubated for $48 \mathrm{~h}$ in polystyrene well-plates in the presence or absence of lipopolysaccharide (LPS) and exposed to different pyrophosphate (PPi) concentrations; CtrlM = control media incubated for $48 \mathrm{~h}$ in polystyrene well-plates in the presence or absence of LPS and exposed to different PPi concentrations; DEM = direct effect media incubated for $48 \mathrm{~h}$ in $50 \mathrm{~mL}$ tubes and used for direct exposure of MSCs to LPS and different PPi concentrations. The different PPi concentrations were as follows: $0 \mu \mathrm{M}$ (PPi0), $50 \mu \mathrm{M}$ (PPi1), $100 \mu \mathrm{M}$ (PPi2) and $250 \mu \mathrm{M}$ (PPi3). The line graph (top) shows the interaction effect of culture condition and exposure to LPS, whereas the bar graph (bottom) shows the mean and standard error of the mean $(n=4-5)$. Connected bars indicate significant differences $(P<0.05 ; P<0.001)$ between LPS and non-LPS as well as between the different conditions (CM, CtrlM, DEM). Small letters indicate significant differences between PPi concentrations (PPi0, PPi1, PPi2, PPi3), where each two similar letters indicate a statistically significant difference $(P<0.05)$ between the two concentrations they are representing

(1.2- to 2-fold downregulation, $P<0.05$ across groups) and between PPi3 and PPi1 (1.2- to 2-fold downregulation,
$P<0.05$ across groups) (Fig. 6a). Additionally, the gene expression of PPAR- $\gamma$, which regulates the differentiation of MSCs into adipocytes, was significantly downregulated 3- to 3.5-fold when MSCs were exposed to inflammatory monocyte-conditioned media compared to nonstimulated monocyte $\mathrm{CM}(P<0.001$ across the material groups) (Fig. $6 \mathrm{~b})$.

\section{Discussion}

The present study investigated the role of monocyte-toMSC communication via soluble factors on bone differentiation in inflammatory and noninflammatory environments in vitro. Furthermore, the effects of pyrophosphate on MSC differentiation, as well as monocyte and MSC survival, were addressed.

\subsection{Effects of LPS and PPi on monocyte adhesion/ cell numbers and viability}

Bacterial stimuli (LPS) had a profound effect on the distribution of monocytes, resulting in higher cell adhesion and a lower number of monocytes in suspension. A similar effect has been observed elsewhere and attributed to reduced caspase activity and increased Akt phosphorylation [35]. In contrast to monocytes, the amount and distribution of MSCs were not affected by direct exposure to LPS. However, the amount of MSCs in the supernatant of conditioned medium obtained from LPS-primed inflammatory monocytes decreased considerably. It is likely that the conditioned media contained inflammatory factors secreted from monocytes exposed to LPS, which promoted MSC viability and adhesion. Interestingly, MSC viability increased significantly in LPS-monocyte-conditioned media. Thus, LPS-stimulated monocytes appear to produce survival factor(s) that improve MSC viability. In contrast, MSCs incubated in noninflammatory monocyteconditioned media (-LPS) exhibited significantly lower viability. It is possible that the lower MSC viability in monocyte-conditioned medium (CM) compared to CtrM and DEM is due to the low starting media serum levels (1\%), which were further reduced during the $48 \mathrm{~h}$ incubation with monocytes. Taking into account the serum depletion occurring in all CM groups, the observed increase in MSC viability in LPS-primed monocyte CM appears even more potent.

\subsection{PPi increases the survival of primary human monocytes and MSCs}

In the present study, cells were exposed to pyrophosphate, both as ions in solution (sodium pyrophosphate) in all three conditions and as precipitants (in the form of amorphous 
(a)
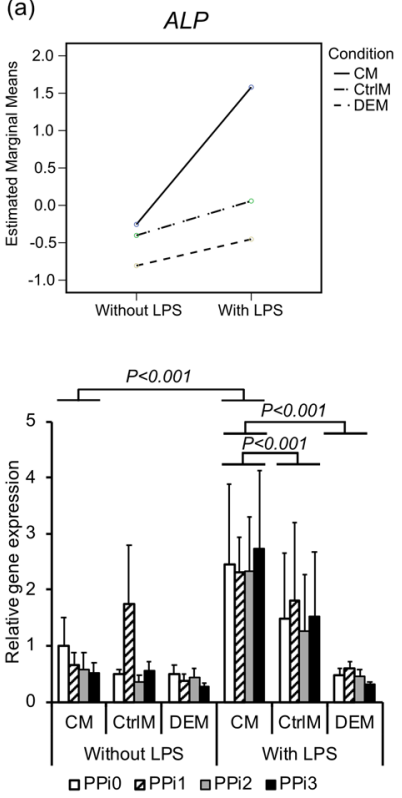

(b)
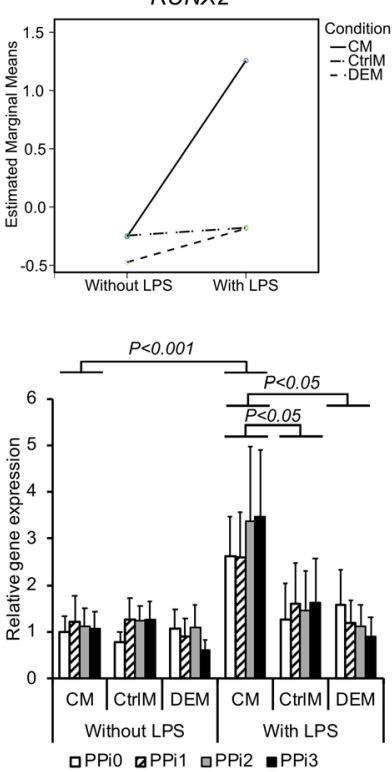

Fig. 5 Gene expression of bone-related factors in mesenchymal stem cells (MSCs). Gene expression analysis of (a) alkaline phosphatase $(A L P),(\mathbf{b})$ runt-related transcription factor $2(R U N X 2),(\mathbf{c})$ collagen 1 alpha 1 (COL1A1) and (d) bone morphogenetic protein $2(B M P-2)$ in MSCs after $72 \mathrm{~h}$ of culture in different culture media: $\mathrm{CM}=$ conditioned media from monocytes incubated for $48 \mathrm{~h}$ in polystyrene well plates in the presence or absence of lipopolysaccharide (LPS) and exposed to different pyrophosphate (PPi) concentrations; $\mathrm{CtrlM}=$ control media incubated for $48 \mathrm{~h}$ in polystyrene well plates in the presence or absence of LPS and exposed to different PPi

calcium pyrophosphate precipitant) [15] in monocyte cultures and under DEM conditions for MSCs. In monocyte cultures, the presence of high PPi concentrations $(250 \mu \mathrm{M})$ significantly increased the number of monocytes in suspension without affecting the number of adherent monocytes. Thus, monocytes exhibited a survival effect from PPi at low-serum levels (1\%), similar to previous reports in fibroblasts [36]. MSC viability (LDH) was higher following exposure to $250 \mu \mathrm{M}$ PPi than following exposure to $0 \mathrm{PPi}$, further supporting a survival factor-like effect of PPi in multiple cell types. The increase in cell viability was particularly apparent under DEM conditions, implicating the PPiprecipitates - present in both monocytic- and DEM-cultures - as responsible for this effect. PPi precipitants require direct contact with the cell membrane [37], as the survival effects of pyrophosphate are abolished if cell cultures are inverted (grown on the ceiling of the culture well), thus indicating that it is the interaction between the PPi crystals and the cell surface that is of importance for increased cell survival. Interestingly, other calcium-containing, basic calcium phosphate (BCP) particles produce survival effects in macrophages [38]. Furthermore, calcium pyrophosphate, known to have low solubility and dissolution properties, produces similar responses in inflammatory cells as BCPs [15]. (c)

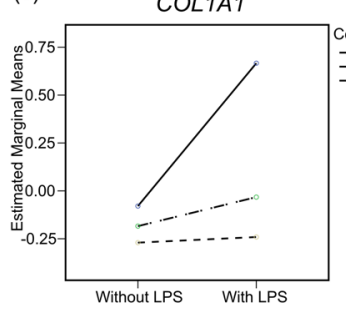

(d)
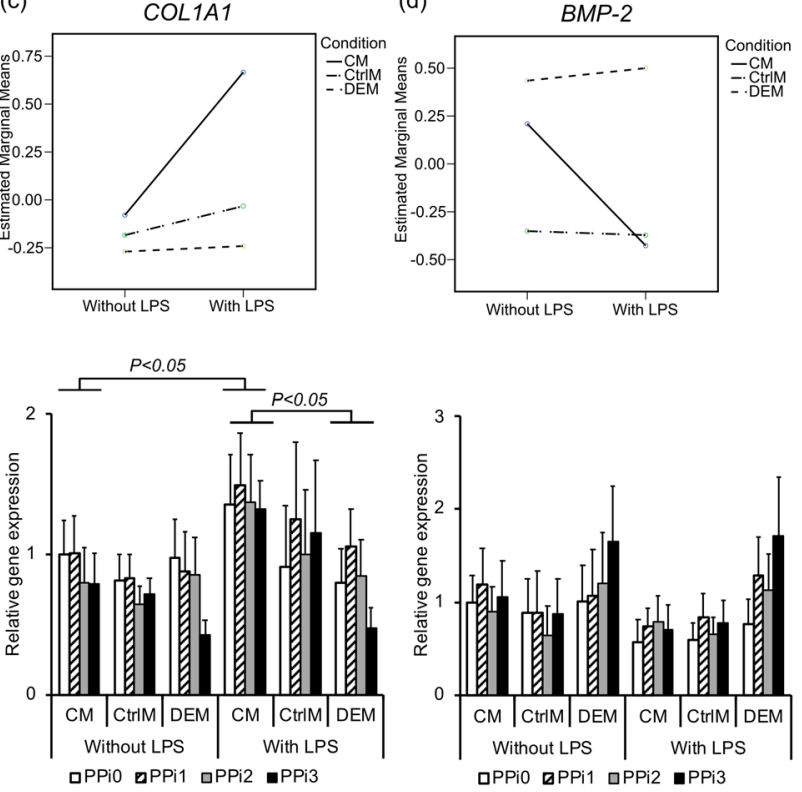

concentrations; $D E M=$ direct effect media incubated for $48 \mathrm{~h}$ in $50 \mathrm{~mL}$ tubes and used for direct exposure of MSCs to LPS and different PPi concentrations. The different PPi concentrations were as follows: $0 \mu \mathrm{M}$ (PPi0), $50 \mu \mathrm{M}$ (PPi1), $100 \mu \mathrm{M}$ (PPi2) and $250 \mu \mathrm{M}$ (PPi3). The line graph (top) shows the interaction effect of culture condition and exposure to LPS, whereas the bar graph (bottom) shows the mean and standard error of the mean $(n=4-5)$. Connected bars indicate significant differences $(P<0.05 ; P<0.001)$ between LPS and non-LPS as well as between the different conditions (CM, CtrlM, DEM)

This is the first study to report that pyrophosphate can act as a survival factor for human MSCs and primary monocytes. Prior studies have investigated the effect of pyrophosphate on the survival of connective tissue cells (fibroblasts) [36] and osteoblasts [15]. The physiological impact of this finding is that PPi may prolong the survival of recruited monocytes, thereby altering both the duration and magnitude of inflammatory signaling. These observations indicate that the role of monocytes and macrophages during bone regeneration induced by pyrophosphate-containing bone ceramics $[16,17,28]$ should be explored.

\subsection{LPS and monocyte-conditioned media promote osteogenic differentiation in MSCs}

A key observation was that monocytes play a role in the differentiation of MSCs, preferentially in inflammatory conditions. The gene expression of RUNX2, ALP and COL1A1 was significantly upregulated in MSCs that were exposed to monocyte-conditioned media (+LPS). The expression of these three genes is necessary for bone cell differentiation and bone tissue formation. In addition, both the cartilage- and adiposecell differentiation pathways (SOX9 and PPAR- $\gamma$ ) were downregulated in response to monocyte-conditioned media (+LPS). 
(a)

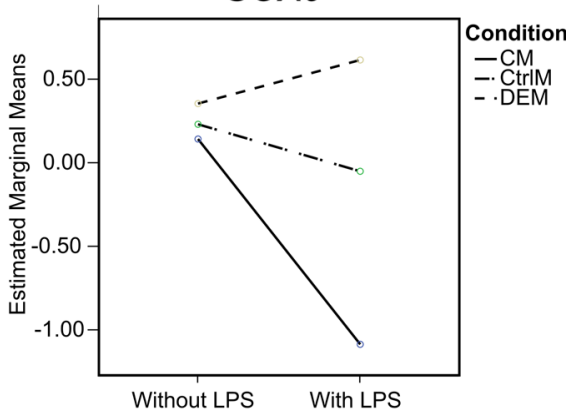

$P<0.001$

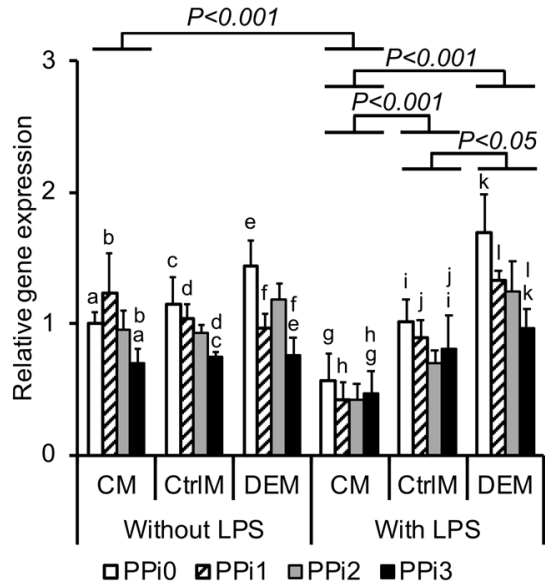

Fig. 6 Gene expression of chondrogenic and adipogenic factors in mesenchymal stem cells (MSCs). Gene expression analysis of (a) chondrogenic transcription factor SRY-box 9 (SOX9) and (b) adipogenic transcription factor peroxisome proliferator-activated receptor gamma $(P P A R-\gamma)$ in MSCs after $72 \mathrm{~h}$ of culture in different culture media: $\mathrm{CM}=$ conditioned media from monocytes incubated for $48 \mathrm{~h}$ in polystyrene well-plates in the presence or absence of lipopolysaccharide (LPS) and exposed to different pyrophosphate (PPi) concentrations; $\mathrm{CtrlM}=$ control media incubated for $48 \mathrm{~h}$ in polystyrene well-plates in the presence or absence of LPS and exposed to different PPi concentrations; DEM $=$ direct effect media incubated for $48 \mathrm{~h}$ in $50 \mathrm{~mL}$ tubes and used for direct exposure of MSCs to LPS and

Monocytes, which differentiate into macrophages, are known to produce multiple cytokines, chemokines, growth factors, and other mediators of inflammation. Macrophages are key players in orchestrating the cascade of events that ultimately resolve inflammation and promote tissue healing. In response to infection (or inflammation), primed monocytes produce factors that direct MSCs towards the bone lineage. In agreement with our findings in vitro, infection and inflammation can potentiate bone formation in vivo [10, 39, 40] Mineralized tissue formation, specifically at the material/tissue interface in vivo, is significantly increased when the implants are preincubated in LPS or when monocytes are selectively polarized (M1, M2) compared to controls [10, 39-41].

The exposure timing, when monocytes sense and react to the environment, is also of importance. Unactivated and M1 macrophages stimulate osteogenic differentiation of MSCs only during the early stages (1-7 days), whereas M2
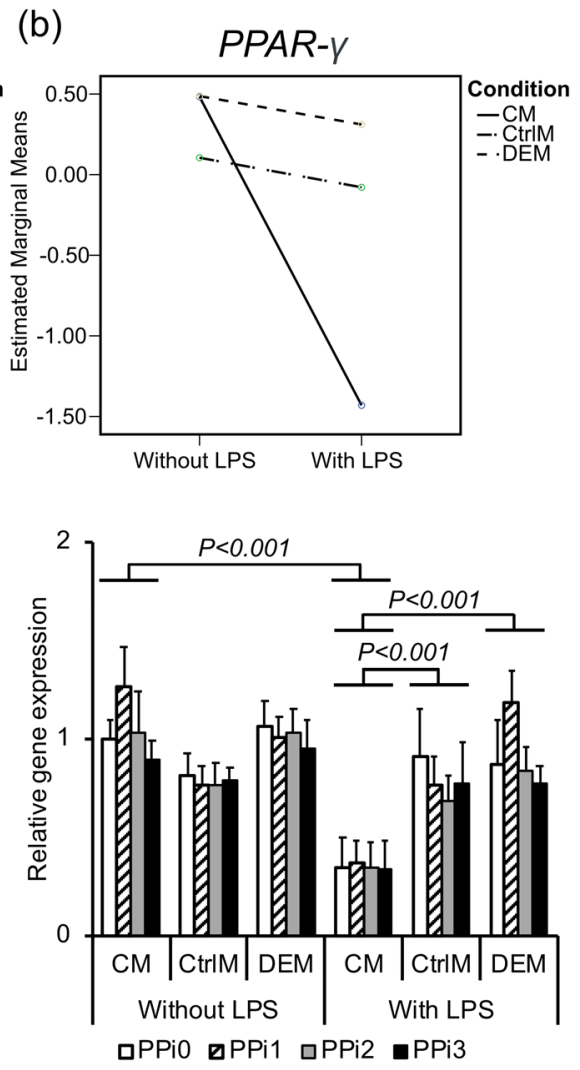

different PPi concentrations. The different PPi concentrations were as follows: $0 \mu \mathrm{M}$ (PPi0), $50 \mu \mathrm{M}$ (PPi1), $100 \mu \mathrm{M}$ (PPi2) and $250 \mu \mathrm{M}$ (PPi3). The line graph (top) shows the interaction effect of culture condition and exposure to LPS, whereas the bar graph (bottom) shows the mean and standard error of the mean $(n=4-5)$. Connected bars indicate significant differences $(P<0.05 ; P<0.001)$ between LPS and non-LPS as well as between the different conditions (CM, CtrlM, DEM). Small letters indicate significant differences between PPi concentrations (PPi0, PPi1, PPi2, PPi3), where every two similar letters indicate a statistically significant difference $(P<0.05)$ between the two concentrations they are representing

polarized macrophages stimulate proliferation and differentiation, including matrix proteins such as osteocalcin and bone sialoprotein, more strongly at late (7-14 days) but not early time points (1-7 days) [39]. Monocytes incubated with LPS, or polarized directly to M1, also secrete molecules that promote increased BMP-2 gene expression in human MSCs [10, 42]. In the present study, a trend for upregulation of BMP-2 gene expression in MSCs was observed only in response to PPi exposure and not in response to monocyte-conditioned media, regardless of the presence of LPS.

\subsection{PPi downregulate SOX9 expression in MSCs}

Interestingly, in MSCs, the expression of only one of the genes analyzed was dependent upon the PPi concentration: SOX9. The expression of SOX9 decreased in response to 
PPi in a concentration-dependent manner. The $S O X 9$ gene is involved in the chondrogenic differentiation of MSCs [43]. Reduced SOX9 expression is implicated in excessive/ectopic mineralization, whereas overexpression of $S O X 9$ is associated with increased chondrogenesis and reduced osteogenesis [43-45]. The concomitant reduction in SOX9 and PPAR- $\gamma$ gene expression strongly favors the differentiation of MSCs towards osteogenic lineages rather than adipogenic or chondrogenic lineages, suggesting that the osteogenic effects of PPi may be due, in part, to a decrease in adipogenic and chondrogenic differentiation signaling. Interestingly, $P P A R-\gamma$ expression and phosphorylation are related to MSC serum levels [46]. Since pyrophosphate can act as a survival factor in low-serum conditions [36], it is possible that pyrophosphate affects gene expression via serum sensing/signaling [15].

The expression of several genes was affected by PPi concentration (although not significantly), particularly following direct exposure to pyrophosphate solution/precipitant (DEM). RUNX2 gene expression was negatively affected by exposure to pyrophosphate, whereas expression of the COL1A1, ALP and TGF- $\beta$ genes was elevated at lower concentrations of PPi but then reduced in a concentrationdependent manner when the PPi concentration was increased. In contrast, $B M P-2$ expression was increased in a concentration-dependent manner when exposed to PPi but only in the DEM group. BMP-2 is a strong inducer of osteogenic differentiation and bone tissue formation, and the apparent concentration-dependent increase in expression in response to pyrophosphate under direct exposure conditions (DEM) further supports the notion that pyrophosphate can elicit osteogenic expression in MSCs.

In prior studies, a biphasic effect on ALP activity and gene expression was observed in preosteoblasts, whereby $100 \mathrm{nM}$ sodium pyrophosphate stimulated greater osteogenic gene expression than $100 \mu \mathrm{M}$ [15]. In the present study, lower concentrations of pyrophosphate also stimulated higher osteogenic gene expression in MSCs (DEM). The optimal concentration in the present study $(50 \mu \mathrm{M})$ was just below the concentration at which sodium pyrophosphate precipitates as calcium pyrophosphate, whereas in osteoblasts, the optimal concentration was much lower (100 nM) [15].

To the authors' knowledge, this is the first study to examine the effect of pyrophosphate on cell-cell communication. Taken together, the results of the present work suggest that pyrophosphate directly stimulates MSCs towards an osteogenic, bone-forming lineage while also discouraging differentiation into fat- and cartilage lineages, but only when in direct contact with crystals/precipitants. These findings are particularly significant because prior studies have focused solely on single cell types or single pathophysiologies (i.e., osteoblast-mediated mineralization or pseudogout), rather than on how pyrophosphate stimulates intercellular communication, leading to inflammatory cell polarization [13], cellular signaling between different cell types [47], and osteogenic differentiation [15].

The findings of the present study refute the underlying null-hypothesis, and show that that PPi conveys direct effects on monocytes and MSCs, increasing their survival. Moreover, the data revealed significant effect of PPi on regulating the early gene expression of MSCs, favoring the downregulation of SOX9 gene that drives the MSCs toward the chondrogenic lineage. Moreover, the present study extends and corroborates previous observations [10] that factors secreted from LPS-stimulated pro-inflammatory macrophages act as potent stimuli for early osteogenic gene expression in the recipient MSCs. The latter finding is also of clinical interest, emphasizing the important role of the initial inflammatory response after surgery and material implantation for triggering the subsequent regenerative processes. In addition, there is mounting evidence that calcium pyrophosphate can enhance bone formation in vivo when mixed with calcium phosphate cement $[16,28]$. We have demonstrated two mechanisms that may contribute to this phenomenon. Pyrophosphate, as an additive or coating, may tentatively improve the biological response to other bioceramic implants. If proven, this would be important for clinical translation, enabling the production and control of the properties of inorganic additives.

It should be noted that a) the media for direct and indirect exposure to pyrophosphate lacked osteogenic factors, such as dexamethasone, ascorbic acid, or beta-glycerol phosphate; and b) the selected time points in the present study were relatively early, excluding observations at later but still relevant time stages for osteogenic differentiation. Without osteogenic media, multipotent cells typically exhibit much higher stochastic "noise," and the absolute magnitude of the cellular response (i.e., changes in gene expression) to external stimulants (i.e., different surface chemistry) is often quite weak compared to cultures that include osteogenic media. Subsequent studies should investigate how PPi affects MSC differentiation under osteogenic conditions, where effects are likely to occur at much greater magnitudes.

\section{Conclusions}

In conclusion, the results of the present in vitro study showed that high concentrations of pyrophosphate improved monocyte and MSC survival. Furthermore, the inflammatory environment, including monocyte-MSC paracrine communication, affected the early differentiation of MSCs towards osteogenesis. Calcium pyrophosphate precipitants stimulated pro-osteogenic gene expression. 
Inflammatory monocytes appear to skew MSCs towards the osteogenic lineage by (1) potentiating MSCs to early differentiation into bone cells via increased expression of RUNX2, ALP and COLIAl and (2) hindering MSC differentiation towards the cartilage and adipocyte lineages, as revealed by suppressed $S O X 9$ and $P P A R-\gamma$ expression.

Acknowledgements This research was funded by grants from the Swedish Foundation for Strategic Research (SSF) (\#RMA15-0110), the Swedish Research Council (2018-02891), the Swedish state under the agreement between the Swedish government and the county councils, the ALF agreement (ALFGBG-725641), the IngaBritt and Arne Lundberg Foundation, the Eivind and Elsa K:son Sylvan Foundation, the Hjalmar Svensson Foundation, and the Area of Advance Materials of Chalmers and GU Biomaterials within the Strategic Research Area initiative launched by the Swedish government. The funders had no role in the design of the study; in the collection, analyses, or interpretation of data; in the writing of the manuscript, or in the decision to publish the results.

Funding Open access funding provided by University of Gothenburg.

\section{Compliance with ethical standards}

Conflict of interest HE has two patents (US20130066324A130 and US9220597B232), is a shareholder and consultant with OssDsign AB, a company which sells calcium phosphate products which include pyrophosphate. PT is a shareholder of OssDsign AB. All other authors declare no competing interests.

Publisher's note Springer Nature remains neutral with regard to jurisdictional claims in published maps and institutional affiliations.

Open Access This article is licensed under a Creative Commons Attribution 4.0 International License, which permits use, sharing, adaptation, distribution and reproduction in any medium or format, as long as you give appropriate credit to the original author(s) and the source, provide a link to the Creative Commons license, and indicate if changes were made. The images or other third party material in this article are included in the article's Creative Commons license, unless indicated otherwise in a credit line to the material. If material is not included in the article's Creative Commons license and your intended use is not permitted by statutory regulation or exceeds the permitted use, you will need to obtain permission directly from the copyright holder. To view a copy of this license, visit http://creativecommons. org/licenses/by/4.0/.

\section{References}

1. Baht GS, Vi L, Alman BA. The role of the immune cells in fracture healing. Curr Osteoporos Rep. 2018; https://doi.org/10. 1007/s11914-018-0423-2.

2. Barradas AM, Monticone V, Hulsman M, Danoux C, Fernandes $\mathrm{H}$, Tahmasebi Birgani $\mathrm{Z}$, et al. Molecular mechanisms of biomaterial-driven osteogenic differentiation in human mesenchymal stromal cells. Integr Biol. 2013; https://doi.org/10.1039/ c3ib40027a.

3. Gomez-Barrena E, Rosset P, Gebhard F, Hernigou P, Baldini N, Rouard $\mathrm{H}$, et al. Feasibility and safety of treating non-unions in tibia, femur and humerus with autologous, expanded, bone marrow-derived mesenchymal stromal cells associated with biphasic calcium phosphate biomaterials in a multicentric, non- comparative trial. Biomaterials. 2019; https://doi.org/10.1016/j. biomaterials.2018.03.033.

4. Shi C, Pamer EG. Monocyte recruitment during infection and inflammation. Nat Rev Immunol. 2011; https://doi.org/10.1038/ nri3070.

5. Cho HH, Bae YC, Jung JS. Role of toll-like receptors on human adipose-derived stromal cells. Stem Cells. 2006; https://doi.org/ 10.1634/stemcells.2006-0189.

6. Bronner F, Farach-Carson MC, Rubin J. Bone resorption. London: Springer; 2005.

7. Longoni A, Knezevic L, Schepers K, Weinans H, Rosenberg A, Gawlitta D. The impact of immune response on endochondral bone regeneration. NPJ Regen Med. 2018; https://doi.org/10. 1038/s41536-018-0060-5.

8. Mountziaris PM, Mikos AG. Modulation of the inflammatory response for enhanced bone tissue regeneration. Tissue Eng Part B Rev. 2008; https://doi.org/10.1089/ten.teb.2008.0038.

9. Mountziaris PM, Spicer PP, Kasper FK, Mikos AG. Harnessing and modulating inflammation in strategies for bone regeneration. Tissue Eng Part B Rev. 2011; https://doi.org/10.1089/ten.TEB. 2011.0182

10. Omar OM, Graneli C, Ekstrom K, Karlsson C, Johansson A, Lausmaa $\mathrm{J}$, et al. The stimulation of an osteogenic response by classical monocyte activation. Biomaterials. 2011; https://doi.org/ 10.1016/j.biomaterials.2011.07.055.

11. Humbert P, Brennan MA, Davison N, Rosset P, Trichet V, Blanchard $\mathrm{F}$, et al. Immune modulation by transplanted calcium phosphate biomaterials and human mesenchymal stromal cells in bone regeneration. Front Immunol. 2019; https://doi.org/10.3389/ fimmu.2019.00663.

12. Wang M, Chen F, Wang J, Chen X, Liang J, Yang X, et al. Calcium phosphate altered the cytokine secretion of macrophages and influenced the homing of mesenchymal stem cells. J Mater Chem B. 2018; https://doi.org/10.1039/c8tb01201f.

13. Campillo-Gimenez L, Renaudin F, Jalabert M, Gras P, Gosset M, Rey $\mathrm{C}$, et al. Inflammatory potential of four different phases of calcium pyrophosphate relies on NF-kappaB activation and MAPK pathways. Front Immunol. 2018; https://doi.org/10.3389/ fimmu.2018.02248.

14. Roch-Arveiller M, Legros R, Chanaud B, Muntaner O, Strzalko S, Thuret A, et al. Inflammatory reactions induced by various calcium pyrophosphate crystals. Biomed Pharmacother. 1990; https://doi.org/10.1016/0753-3322(90)90207-p.

15. Pujari-Palmer M, Pujari-Palmer S, Lu X, Lind T, Melhus H, Engstrand T, et al. Pyrophosphate stimulates differentiation, matrix gene expression and alkaline phosphatase activity in osteoblasts. PLoS One. 2016; https://doi.org/10.1371/journal. pone. 0163530 .

16. Engstrand T, Kihlstrom L, Lundgren K, Trobos M, Engqvist H, Thomsen P. Bioceramic implant induces bone healing of cranial defects. Plast Reconstr Surg Glob Open. 2015; https://doi.org/10. 1097/gox.0000000000000467.

17. Grover LM, Wright AJ, Gbureck U, Bolarinwa A, Song J, Liu Y, et al. The effect of amorphous pyrophosphate on calcium phosphate cement resorption and bone generation. Biomaterials. 2013; https://doi.org/10.1016/j.biomaterials.2013.05.001.

18. Li Y, Niu S, Xi D, Zhao S, Sun J, Jiang Y, et al. Differences in lipopolysaccharides-induced inflammatory response between mouse embryonic fibroblasts and bone marrow-derived macrophages. J Interferon Cytokine Res. 2019; https://doi.org/10.1089/ jir.2018.0167.

19. Lu YC, Yeh WC, Ohashi PS. LPS/TLR4 signal transduction pathway. Cytokine. 2008; https://doi.org/10.1016/j.cyto.2008. 01.006 .

20. Ezura Y, Lin X, Hatta A, Izu Y, Noda M. Interleukin-1beta suppresses the transporter genes Ank and Ent1 expression in 
stromal progenitor cells retaining mineralization. Calcif Tissue Int. 2016; https://doi.org/10.1007/s00223-016-0139-1.

21. Minashima T, Quirno M, Lee YJ, Kirsch T. The role of the progressive ankylosis protein (ANK) in adipogenic/osteogenic fate decision of precursor cells. Bone. 2017; https://doi.org/10.1016/j. bone.2017.03.003.

22. Roberts F, Zhu D, Farquharson C, Macrae VE. ENPP1 in the regulation of mineralization and beyond. Trends Biochem Sci. 2019; https://doi.org/10.1016/j.tibs.2019.01.010.

23. Villa-Bellosta R, Rivera-Torres J, Osorio FG, Acin-Perez R, Enriquez JA, Lopez-Otin C, et al. Defective extracellular pyrophosphate metabolism promotes vascular calcification in a mouse model of Hutchinson-Gilford progeria syndrome that is ameliorated on pyrophosphate treatment. Circulation. 2013; https://doi.org/10.1161/ circulationaha.112.000571.

24. Kozelskaya AI, Bolbasov EN, Golovkin AS, Mishanin AI, Viknianshchuk AN, Shesterikov EV, et al. Modification of the ceramic implant surfaces from zirconia by the magnetron sputtering of different calcium phosphate targets: a comparative study. Materials (Basel). 2018; https://doi.org/10.3390/ma11101949.

25. Bouchard L, de Médicis R, Lussier A, Naccache PH, Poubelle PE. Inflammatory microcrystals alter the functional phenotype of human osteoblast-like cells in vitro: synergism with IL-1 to overexpress cyclooxygenase-2. J Immunol. 2002; https://doi.org/ 10.4049/jimmunol.168.10.5310.

26. Lee JH, Ryu HS, Lee DS, Hong KS, Chang BS, Lee CK. Biomechanical and histomorphometric study on the bone-screw interface of bioactive ceramic-coated titanium screws. Biomaterials. 2005; https://doi.org/10.1016/j.biomaterials.2004.08.033.

27. Takahashi K, van den Beucken JJ, Wolke JG, Hayakawa T, Nishiyama N, Jansen JA. Characterization and in vitro evaluation of biphasic calcium pyrophosphate-tricalciumphosphate radio frequency magnetron sputter coatings. J Biomed Mater Res A. 2008; https://doi.org/10.1002/jbm.a.31341.

28. Omar O, Engstrand T, Kihlström Burenstam Linder L, Åberg J, Shah FA, Palmquist A, et al. In situ bone regeneration of large cranial defects using synthetic ceramic implants with a tailored composition and design. Proc Natl Acad Sci USA. 2020; https:// doi.org/10.1073/pnas.2007635117.

29. Hulsart-Billstrom G, Dawson JI, Hofmann S, Muller R, Stoddart MJ, Alini M, et al. A surprisingly poor correlation between in vitro and in vivo testing of biomaterials for bone regeneration: results of a multicentre analysis. Eur Cell Mater. 2016; https://doi. org/10.22203/ecm.v031a20.

30. Sivolella S, Brunello G, Ferroni L, Berengo M, Meneghello R, Savio G, et al. A novel in vitro technique for assessing dental implant osseointegration. Tissue Eng Part C Methods. 2016; https:// doi.org/10.1089/ten.TEC.2015.0158.

31. Sladkova-Faure M, Pujari-Palmer M, Öhman-Mägi C, López A, Wang $\mathrm{H}$, Jr., Engqvist $\mathrm{H}$, et al. A biomimetic engineered bone platform for advanced testing of prosthetic implants. Sci Rep. 2020; https://doi.org/10.1038/s41598-020-78416-w.

32. Su J, Du Z, Xiao L, Wei F, Yang Y, Li M, et al. Graphene oxide coated titanium surfaces with osteoimmunomodulatory role to enhance osteogenesis. Mater Sci Eng C Mater Biol Appl. 2020; https://doi.org/10.1016/j.msec.2020.110983.

33. Kazimierczak P, Koziol M, Przekora A. The Chitosan/Agarose/ NanoHA bone scaffold-induced M2 macrophage polarization and its effect on osteogenic differentiation in vitro. Int $\mathrm{J}$ Mol Sci. 2021; https://doi.org/10.3390/ijms22031109.

34. Lennerås M, Ekström K, Vazirisani F, Shah FA, Junevik K, Thomsen $\mathrm{P}$, et al. Interactions between monocytes, mesenchymal stem cells, and implants evaluated using flow cytometry and gene expression. J Tissue Eng Regen Med. 2018; https://doi.org/10. $1002 /$ term. 2700

35. Goyal A, Wang Y, Graham MM, Doseff AI, Bhatt NY, Marsh CB. Monocyte survival factors induce Akt activation and suppress caspase-3. Am J Respir Cell Mol Biol. 2002; https://doi.org/10. 1165/ajrcmb.26.2.4640.

36. Rubin AH, Bowen-Pope DF. Coordinate control of Balb/c3T3 cell survival and multiplication by serum or calcium pyrophosphate complexes. J Cell Physiol. 1979; https://doi.org/10.1002/jcp. 1040980110.

37. Renaudin F, Sarda S, Campillo-Gimenez L, Severac C, Leger T, Charvillat $\mathrm{C}$, et al. Adsorption of proteins on $\mathrm{m}-\mathrm{CPPD}$ and urate crystals inhibits crystal-induced cell responses: study on albumincrystal interaction. J Funct Biomater. 2019; https://doi.org/10. 3390/jfb10020018.

38. Hamilton JA, McCarthy G, Whitty G. Inflammatory microcrystals induce murine macrophage survival and DNA synthesis. Arthritis Res. 2001; https://doi.org/10.1186/ar308.

39. Sridharan R, Cameron AR, Kelly DJ, Kearney CJ, O'Brien FJ. Biomaterial based modulation of macrophage polarization: a review and suggested design principles. Materials Today. 2015; https://doi.org/10.1016/j.mattod.2015.01.019.

40. Zhang Y, Bose T, Unger RE, Jansen JA, Kirkpatrick CJ, van den Beucken J. Macrophage type modulates osteogenic differentiation of adipose tissue MSCs. Cell Tissue Res. 2017; https://doi.org/10. 1007/s00441-017-2598-8.

41. Ma QL, Zhao LZ, Liu RR, Jin BQ, Song W, Wang Y, et al. Improved implant osseointegration of a nanostructured titanium surface via mediation of macrophage polarization. Biomaterials. 2014; https://doi.org/10.1016/j.biomaterials.2014.08.025.

42. Ekstrom K, Omar O, Graneli C, Wang X, Vazirisani F, Thomsen P. Monocyte exosomes stimulate the osteogenic gene expression of mesenchymal stem cells. PLoS One. 2013; https://doi.org/10. 1371/journal.pone.0075227.

43. Liao J, Hu N, Zhou N, Lin L, Zhao C, Yi S, et al. Sox9 potentiates BMP2-induced chondrogenic differentiation and inhibits BMP2induced osteogenic differentiation. PLoS One. 2014; https://doi. org/10.1371/journal.pone.0089025.

44. Loebel C, Czekanska EM, Bruderer M, Salzmann G, Alini M, Stoddart MJ. In vitro osteogenic potential of human mesenchymal stem cells is predicted by Runx2/Sox9 ratio. Tissue Eng Part A. 2015; https://doi.org/10.1089/ten.TEA.2014.0096.

45. Peacock JD, Levay AK, Gillaspie DB, Tao G, Lincoln J. Reduced sox9 function promotes heart valve calcification phenotypes in vivo. Circ Res. 2010; https://doi.org/10.1161/circresaha.109.213702.

46. Wu L, Cai X, Dong H, Jing W, Huang Y, Yang X, et al. Serum regulates adipogenesis of mesenchymal stem cells via MEK/ ERK-dependent PPARgamma expression and phosphorylation. J Cell Mol Med. 2010; https://doi.org/10.1111/j.1582-4934. 2009.00709.x.

47. McCarthy GM, Dunne A. Calcium crystal deposition diseases beyond gout. Nat Rev Rheumatol. 2018; https://doi.org/10.1038/ s41584-018-0078-5. 